\title{
SECOND KIND CHEBYSHEV WAVELET METHOD FOR SOLVING SYSTEM OF LINEAR DIFFERENTIAL EQUATIONS
}

\author{
Pammy Manchanda ${ }^{1}$, Mamta Rani $^{2} \S$ \\ ${ }^{1,2}$ Department of Mathematics \\ Guru Nanak Dev University \\ Amritsar, 143005, INDIA
}

\begin{abstract}
Wavelet methods have been used extensively for the solution of various problems of science and engineering. In this paper, we attempt to solve system of linear differential equations by second kind Chebyshev wavelet method. The key idea of this approach is that it reduces the underlying problem to a system of algebraic equations. Illustrative examples are included to demonstrate the efficiency and accuracy of the proposed method. The numerical solutions of system of differential equations occurring in drug therapy of irregular heartbeats and pond pollution are obtained by using this method.
\end{abstract}

AMS Subject Classification: 42C40, 42A38, 65M99

Key Words: second kind Chebyshev wavelets, operational matrix of integration, system of linear differential equations

\section{Introduction}

Wavelet analysis has become an important tool for mathematicians, physicists, and engineers to study real world problems modeled by differential equations.

Since some of these problems do not have exact solution, numerical methods are used to solve them. Many authors have used wavelet related numerical and

\footnotetext{
Received: December 20, 2016

Revised: $\quad$ February 2, 2017

Published:

April 20, 2017

$\S_{\text {Correspondence author }}$
}

(c) 2017 Academic Publications, Ltd. url: www.acadpubl.eu 
approximate methods to solve differential equations see ([7] and [8]), integral equations [9], variational problems [10] etc.

Several techniques such as Adomain decomposition method [3], Variational iteration method [6], differential transformation method [5], combined Laplace Transfom - Adomain Decomposition Method [4] have been used for solving system of linear differential equations. We present a numerical method to solve system of linear differential equations based on second kind Chebyshev wavelet. Babolian and Fattahzadeh [1] have used Chebyshev Wavelet for numerical solution of differential equations. Celik [14] have applied Chebyshev wavelet collocation method for the numerical solution of Burgers-Huxley equation. Wang et al. [13] used second kind Chebyshev wavelet based approximation method to solve integral equations. Zhu et al. have implemented this method to solve problems of fractional nonlinear Fredholm integro-differential equations and variational problems see([11] and [12]). Wang and Fan [2] have solved fractional differential equations using second kind Chebyshev wavelet.

The method is based upon the second kind Chebyshev wavelets approximation of highest order derivatives of unknown functions. The lower order derivatives and functions are obtained through integration. The Chebyshev wavelet operational matrix of integration is used to reduce the problem to a system of linear differntial equations. The solution can be found by determining the unknown coefficients that satisfy the algebraic equations.

The outline of the paper is as follows: In Section 2 we describe the basic properties of second kind Chebyshev wavelets, function approximation in terms of second kind Chebyshev wavelet and operational matrix of integration. In Section 3 the mathematical formulation of proposed method is given. In Section 4 some examples are solved using the proposed numerical method. Conclusion is drawn in Section 5.

\section{Second Kind Chebyshev Wavelet}

The second kind Chebyshev wavelet $\psi_{n, m}(t)=\psi(k, n, m, t)$ have four arguments $n=1,2, \ldots 2^{k-1}, k$ can assume any positive integer, $\mathrm{m}$ is the degree of the second kind Chebyshev polynomials and $t$ denotes the time. They are defined on the interval $[0,1)$ as

$$
\psi_{n, m}(t)= \begin{cases}2^{\frac{k}{2}} \widetilde{U_{m}}\left(2^{k} t-2 n+1\right) & \text { if } \frac{n-1}{2^{k}-1} \leq t<\frac{n}{2^{k}-1} \\ 0 & \text { otherwise }\end{cases}
$$


where $\widetilde{U_{m}}=\sqrt{\frac{2}{\pi}} U_{m}(t)$ and $m=0,1,2, \ldots, M-1 . U_{m}$ are Chebyshev polynomials of second kind of degree $m$ which are orthogonal with respect to the weight function $w(t)=\sqrt{1-t^{2}}$ on the interval $[-1,1]$. These polynomials satisfy the following recursive formula

$$
\begin{aligned}
U_{0}(t) & =1 \\
U_{1}(t) & =2 t \\
U_{m+1}(t) & =2 t U_{m}(t)-U_{m-1}(t), \quad m=1,2, \ldots \ldots
\end{aligned}
$$

In second kind Chebyshev wavelets the weight function $w(x)$ have to be dilated and translated as $w_{n}(t)=w\left(2^{k} t-2 n+1\right)$ to get orthogonal wavelets.

\subsection{Function Approximation in Terms of Second Kind Chebyshev Wavelet}

Any square integrable function $y(t)$ defined over $[0,1)$ can be expanded by second kind Chebyshev wavelets $\psi_{n m}$ as:

$$
y(t)=\sum_{n=1}^{\infty} \sum_{m=0}^{\infty} c_{n m} \psi_{n m}(t)
$$

where $c_{n m}=\left(y(t), \psi_{n m}(t)\right)$ denotes the inner product. If the infinite series in (2) is truncated, then (2) can be written as

$$
y(t)=\sum_{n=1}^{2^{k-1}} \sum_{m=0}^{M-1} c_{n m} \psi_{n m}(t)=C^{T} \Psi(t),
$$

where $C$ and $\Psi(t)$ are $2^{k-1} M \times 1$ matrices given by

$$
\begin{aligned}
C & =\left\{c_{10}, c_{11}, . ., c_{1(M-1)}, c_{20}, c_{21}, . ., c_{2(M-1)}, c_{2^{k-1} 0}, c_{2^{k-1} 1}, . ., c_{2^{k-1}(M-1)}\right\}^{T}, \quad \text { and } \\
\Psi(t) & =\left\{\psi_{10}, \psi_{11}, . ., \psi_{1(M-1)}, \psi_{20}, \psi_{21}, . ., \psi_{2(M-1)}, \psi_{2^{k-1} 0}, \psi_{2^{k-1} 1}, . ., \psi_{2^{k-1}(M-1)}\right\}^{T} .
\end{aligned}
$$

\subsection{Operational Matrix of Integration}

In this section we introduce operational matrix $P$ of integration for $M=3$, $k=2$ which plays a significant role in solving system of differential equations. 
In this case the six basis functions are given by

$$
\left.\begin{array}{l}
\psi_{10}(t)=2 \sqrt{\frac{2}{\pi}} \\
\psi_{11}(t)=2 \sqrt{\frac{2}{\pi}}(8 t-2) \quad \\
\psi_{12}(t)=2 \sqrt{\frac{2}{\pi}}\left(64 t^{2}-32 t+3\right)
\end{array}\right\} 0 \leq t<\frac{1}{2}
$$

By integrating (3) and (4) from 0 to $t$, we obtain

$$
\begin{gathered}
\int_{0}^{t} \psi_{10}\left(t^{\prime}\right) d t^{\prime}=\left\{\begin{array}{ll}
2 \sqrt{\frac{2}{\pi}} t & , 0 \leq t<\frac{1}{2} \\
\sqrt{\frac{2}{\pi}} & , \frac{1}{2} \leq t<1
\end{array}=\frac{1}{4} \psi_{10}(t)+\frac{1}{8} \psi_{11}(t)+\frac{1}{2} \psi_{20}(t)\right. \\
\int_{0}^{t} \psi_{11}\left(t^{\prime}\right) d t^{\prime}=\left\{\begin{array}{ll}
4 \sqrt{\frac{2}{\pi}}\left(2 t^{2}-1\right) & , 0 \leq t<\frac{1}{2} \\
0 & , \frac{1}{2} \leq t<1
\end{array}=-\frac{3}{16} \psi_{10}(t)+\frac{1}{16} \psi_{12}(t)\right.
\end{gathered}
$$

similarly

$$
\begin{aligned}
& \int_{0}^{t} \psi_{12}\left(t^{\prime}\right) d t^{\prime}=\frac{1}{12} \psi_{10}(t)-\frac{1}{24} \psi_{11}(t) \\
& \int_{0}^{t} \psi_{20}\left(t^{\prime}\right) d t^{\prime}=\frac{1}{4} \psi_{20}(t)+\frac{1}{8} \psi_{21}(t) \\
& \int_{0}^{t} \psi_{21}\left(t^{\prime}\right) d t^{\prime}=-\frac{3}{16} \psi_{20}(t)+\frac{1}{16} \psi_{22}(t) \\
& \int_{0}^{t} \psi_{22}\left(t^{\prime}\right) d t^{\prime}=\frac{1}{12} \psi_{20}(t)-\frac{1}{24} \psi_{21}(t)
\end{aligned}
$$

Thus

$$
\int_{0}^{t} \Psi_{6}\left(t^{\prime}\right) d t^{\prime}=P_{6 \times 6} \Psi_{6}(t)
$$


where $\Psi_{6}(t)=\left[\psi_{10}, \psi_{11}, \psi_{12}, \psi_{20}, \psi_{21}, \psi_{22}\right]^{T}$ and

$$
P=\frac{1}{4}\left[\begin{array}{cccccc}
1 & \frac{1}{2} & 0 & 2 & 0 & 0 \\
\frac{-3}{4} & 0 & \frac{1}{4} & 0 & 0 & 0 \\
\frac{1}{3} & \frac{-1}{6} & 0 & 0 & 0 & 0 \\
0 & 0 & 0 & 1 & \frac{1}{2} & 0 \\
0 & 0 & 0 & \frac{-3}{4} & 0 & \frac{1}{4} \\
0 & 0 & 0 & \frac{1}{3} & \frac{-1}{6} & 0
\end{array}\right]
$$

This matrix can be written as

$$
P=\frac{1}{4}\left[\begin{array}{ll}
L_{3 \times 3} & S_{3 \times 3} \\
O_{3 \times 3} & L_{3 \times 3}
\end{array}\right]
$$

where

$$
L=\left[\begin{array}{ccc}
1 & \frac{1}{2} & 0 \\
-\frac{3}{4} & 0 & \frac{1}{4} \\
\frac{1}{3} & -\frac{1}{6} & 0
\end{array}\right]
$$

and

$$
S=\left[\begin{array}{lll}
2 & 0 & 0 \\
0 & 0 & 0 \\
0 & 0 & 0
\end{array}\right]
$$

In general $\int_{0}^{t} \Psi\left(t^{\prime}\right) d t^{\prime}=P \Psi(t)$ where $P$ is $2^{k-1} M \times 2^{k-1} M$ operational matrix of integration given by

$$
P=\frac{1}{2^{k}}\left[\begin{array}{cccccc}
L & S & S & \cdots & S & S \\
0 & L & S & \cdots & S & S \\
\vdots & \vdots & \vdots & \ddots & \vdots & \vdots \\
O & O & O & \cdots & L & S \\
O & O & O & \cdots & O & L
\end{array}\right]
$$

where $L$ and $S$ are $M \times M$ matrices given by

$$
L=\frac{1}{2^{k}}\left[\begin{array}{ccccc}
1 & \frac{1}{2} & 0 & \cdots & 0 \\
\frac{-3}{4} & 0 & \frac{1}{4} & \cdots & 0 \\
\frac{1}{3} & \frac{-1}{6} & 0 & \cdots & 0 \\
\frac{-1}{4} & 0 & -\frac{1}{8} & \cdots & 0 \\
\vdots & \vdots & \vdots & \ddots & \vdots \\
(-1)^{M-2} \frac{1}{M-1} & 0 & 0 & \cdots & \frac{1}{2(M-1)} \\
(-1)^{M-1} \frac{1}{M} & 0 & 0 & \cdots & 0
\end{array}\right]
$$




$$
S=\left[\begin{array}{cccc}
2 & 0 & \cdots & 0 \\
0 & 0 & \cdots & 0 \\
\vdots & \vdots & \ddots & \vdots \\
0 & 0 & \cdots & 0
\end{array}\right]
$$

Generally, the operational matrix $P^{n}$ of $n$ times integration of $\Psi(t)$ is given by

$$
\underbrace{\int_{0}^{t} \ldots \int_{0}^{t}}_{n-\text { times }} \Psi(t) d t \ldots d t=P^{n} \Psi(t)
$$

\section{Mathematical Formulation}

Consider the following system of linear differential equations

$$
\begin{aligned}
y_{1}^{\prime}(t) & =f_{1}\left(t, y_{1}, y_{2}, \ldots, y_{n}\right) \\
y_{2}^{\prime}(t) & =f_{2}\left(t, y_{1}, y_{2}, \ldots, y_{n}\right) \\
y_{3}^{\prime}(t) & =f_{3}\left(t, y_{1}, y_{2}, \ldots, y_{n}\right) \\
& \vdots \\
y_{n}^{\prime}(t) & =f_{n}\left(t, y_{1}, y_{2}, \ldots, y_{n}\right)
\end{aligned}
$$

where $y_{j}^{\prime}(j=1,2,3, \ldots, n)$ represents the derivative of $y_{j}$ w.r.t. $t$, subject to the initial conditions $y_{1}(0)=c_{1}, y_{2}(0)=c_{2}, \ldots, y_{n}(0)=c_{n}$.

Now we approximate $y_{j}^{\prime}(t)$ in terms of second kind Chebyshev wavelet as follows

$$
y_{j}^{\prime}(t)=C_{j}^{T} \Psi(t)
$$

where $C_{j}^{T}$ are $2^{k-1} M \times 1$ matrices.

Integrating above equation w.r.t $t$ from 0 to $t$, we get

$$
y_{j}(t)=C_{j}^{T} P \Psi(t)+y_{j}(0) d^{T} \Psi(t)
$$

where $d^{T} \Psi(t)$ is the function approximation of 1 in terms of second kind Chebyshev wavelet given by $1=\frac{\sqrt{\pi}}{2 \sqrt{2}}\left[\begin{array}{llllll}1 & 0 & 0 & 1 & 0 & 0\end{array}\right] \Psi(t)=d^{T} \Psi(t)$.

Using above equations into given system (5) we get the algebraic form of system of linear differential equations. On solving these equations we get the values of $C_{j}^{T}$. By substituting value of $C_{j}^{T}$ in above equation we get the value of $y_{j}(t)$. 


\section{Numerical Examples}

Example 1. (Irregular Heartbeats and Lidocaine) In human, lidocaine injection is used for the treatment of irregular heartbeat. A differential equation model for the dynamics of the drug therapy uses:

$x(t)=$ amount of lidocaine in the bloodstream,

$y(t)=$ amount of lidocaine in body tissue.

For a specific body weight, this problem can be written as a following set of differential equations

$$
\begin{aligned}
& x^{\prime}(t)=-0.09 x(t)+0.038 y(t) \\
& y^{\prime}(t)=0.066 x(t)-0.038 y(t)
\end{aligned}
$$

The physically significant initial data is zero drug in the bloodstream $x(0)=0$ and injection dosage $y(0)=y_{0}$. For $y_{0}=1$ the analytic solution of the above system of differential equations is given by

$$
\begin{array}{r}
x(t)=-0.3367 e^{-0.1204 t}+0.3367 e^{-0.0076 t} \\
y(t)=0.2696 e^{-0.1204 t}+0.7304 e^{-0.0076 t}
\end{array}
$$

For numerical solution we use the following function approximation of $x^{\prime}(t)$, $y^{\prime}(t), z^{\prime}(t)$ in terms of second kind Chebyshev wavelet

$$
\begin{aligned}
& x^{\prime}(t)=C_{1}^{T} \Psi(t) \\
& y^{\prime}(t)=C_{2}^{T} \Psi(t)
\end{aligned}
$$

Integrating from 0 to $\mathrm{t}$ using operational matrix of integration, we obtain

$$
\begin{aligned}
& x(t)=C_{1}^{T} P \Psi(t) \\
& y(t)=C_{2}^{T} P \Psi(t)+d^{T} \Psi(t)
\end{aligned}
$$

Given system of differential equations can be written as

$$
\begin{aligned}
C_{1}^{T}(I+0.09 P) \Psi(t)-0.038 C_{2}^{T} P \Psi(t) & =0.038 d^{T} \Psi(t) \\
C_{2}^{T}(I+0.038 P) \Psi(t)-0.066 C_{1}^{T} P \Psi(t) & =-0.038 d^{T} \Psi(t)
\end{aligned}
$$

The above equations are satisfied for each $t$ in the defined interval. Therefore

$$
C_{1}^{T}(I+0.09 P)-0.038 C_{2}^{T} P=0.038 d^{T}
$$


Table 1: Absolute error of exact and approximate solution

\begin{tabular}{|c|c|c|c|c|}
\hline$t$ & $\mid x$ (exact)-x(SCW) $\mid$ & $\mid x$ (exact)-x(Leg.) $\mid$ & $\mid y$ (exact)-y(SCW) $\mid$ & $\mid y$ (exact)-y(Leg.) $\mid$ \\
\hline 0 & 0.00000059540163 & 0.000026848196 & 0.00000074288804 & 0.000033021894 \\
\hline .1 & 0.0000011982515 & 0.000028593088 & 0.000001883761 & 0.000031820179 \\
\hline .2 & 0.0000023183654 & 0.000029665013 & 0.0000038596751 & 0.00002978414 \\
\hline .3 & 0.0000032273899 & 0.000030526423 & 0.0000060936304 & 0.000027490774 \\
\hline .4 & 0.0000043822441 & 0.000031634234 & 0.0000080155336 & 0.000025510177 \\
\hline .5 & 0.0000056737499 & 0.000030934591 & 0.0000097615834 & 0.000021279798 \\
\hline .6 & 0.0000074970207 & 0.000032719068 & 0.000010838924 & 0.000020154065 \\
\hline .7 & 0.0000086855217 & 0.000033869231 & 0.000012702931 & 0.000018242233 \\
\hline .8 & 0.0000096747021 & 0.00003482053 & 0.000014810325 & 0.000016087584 \\
\hline .9 & 0.000010894804 & 0.000036003205 & 0.000016624327 & 0.000014226897 \\
\hline 1 & 0.000012770922 & 0.000037842354 & 0.000017614584 & 0.000013190524 \\
\hline
\end{tabular}

$$
C_{2}^{T}(I+0.038 P)-0.066 C_{1}^{T} P=-0.038 d^{T}
$$

From these equations we get the vectors $C_{1}^{T}, C_{2}^{T}, C_{3}^{T}$. After finding the vectors $C_{1}^{T}, C_{2}^{T}, C_{3}^{T}$ we can get the approximate values of $x(t), y(t), z(t)$ by inserting $C_{1}^{T}, C_{2}^{T}, C_{3}^{T}$ into (6). The absolute error of exact and approximate solution for $M=3, k=2$ using second kind Chebyshev wavelet method(SCW) and Legendre (Leg.) wavelet method is given in Table 1.

Example 2 (Pond Pollution)Consider three ponds connected by streams. The first pond has a pollution source which spreads to the other ponds. Let $g(t)$ denote the pollutant flow rate into pond 1 and $g_{1}, g_{2}, g_{3}$ denote the pollutant flow rates out of ponds 1,2 and 3 respectively. The volume of three ponds is $V_{1}$, $V_{2}, V_{3}$ which remain constant. $x(t), y(t), z(t)$ denote the amount of pollutant in ponds $1,2,3$, respectively. For a particular case we take $\frac{g_{i}}{V_{i}}=0.001$ for $i=1,2,3$ and $g(t)=0.125 \mathrm{lb} / \mathrm{min}$ for the first 48 hours. This problem of pond pollution gives the following system of differential equations

$$
\begin{aligned}
x^{\prime}(t) & =0.001 z(t)-0.001 x(t)+0.125 \\
y^{\prime}(t) & =0.001 x(t)-0.001 y(t) \\
z^{\prime}(t) & =0.001 y(t)-0.001 z(t)
\end{aligned}
$$

with the initial conditions given by

$$
x(0)=y(0)=z(0)=0 .
$$


The analytic solution of this system is given by

$$
\begin{aligned}
& x(t)=e^{\frac{-3 t}{2000}}\left(\frac{125 \sqrt{3}}{9} \sin \left(\frac{\sqrt{3} t}{2000}\right)-\frac{125}{3} \cos \left(\frac{\sqrt{3} t}{2000}\right)\right)+\frac{125}{3}+\frac{t}{24} \\
& y(t)=-\frac{250 \sqrt{3}}{9} e^{\frac{-3 t}{2000}} \sin \left(\frac{\sqrt{3} t}{2000}\right)+\frac{t}{24} \\
& z(t)=e^{\frac{-3 t}{2000}}\left(\frac{125}{3} \cos \left(\frac{\sqrt{3} t}{2000}\right)+\frac{125 \sqrt{3}}{9} \sin \left(\frac{\sqrt{3} t}{2000}\right)\right)-\frac{125}{3}+\frac{t}{24}
\end{aligned}
$$

For numerical solution we use the following function approximation of $x^{\prime}(t)$, $y^{\prime}(t), z^{\prime}(t)$ in terms of second kind Chebyshev wavelet

$$
\begin{aligned}
& x^{\prime}(t)=C_{1}^{T} \Psi(t) \\
& y^{\prime}(t)=C_{2}^{T} \Psi(t) \\
& z^{\prime}(t)=C_{3}^{T} \Psi(t)
\end{aligned}
$$

Integrating from 0 to $t$ using operational matrix of integration, we obtain

$$
\begin{aligned}
& x(t)=C_{1}^{T} P \Psi(t) \\
& y(t)=C_{2}^{T} P \Psi(t) \\
& z(t)=C_{3}^{T} P \Psi(t)
\end{aligned}
$$

Using the above results in given system, we get

$$
\begin{aligned}
& C_{1}^{T}(I+.001 P) \Psi(t)-.001 C_{3}^{T} P \Psi(t)=0.125 d^{T} \Psi(t) \\
& C_{2}^{T}(I+.001 P) \Psi(t)-.001 C_{1}^{T} P \Psi(t)=0 \\
& C_{3}^{T}(I+.001 P) \Psi(t)-.001 C_{2}^{T} P \Psi(t)=0
\end{aligned}
$$

The above equations are satisfied for each $t$ in the defined interval. Therefore

$$
\begin{aligned}
& C_{1}^{T}(I+.001 P)-.001 C_{3}^{T} P=0.125 d^{T} \\
& C_{2}^{T}(I+.001 P)-.001 C_{1}^{T} P=0 \\
& C_{3}^{T}(I+.001 P)-.001 C_{2}^{T} P=0
\end{aligned}
$$

After finding the vectors $C_{1}^{T}, C_{2}^{T}, C_{3}^{T}$ from above system of equations we can get the approximate values of $x(t), y(t), z(t)$. The absolute error of exact and approximate solution for $M=3, k=2$ using second kind Chebyshev wavelet method and Legendre wavelet method is given in Table 2.

Example 3. Consider the following system of linear differential equations

$$
x^{\prime}(t)+y^{\prime}(t)=2 z(t)
$$




\begin{tabular}{|c|c|c|c|c|c|c|c|c|c|c|c|}
\hline$\bullet$ & 6 & $\infty$ & 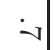 & $\dot{\sigma}$ & cr & $i$ & ن ن & iv & $i$ & o & + \\
\hline 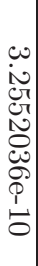 & 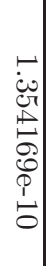 & 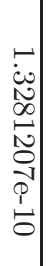 & 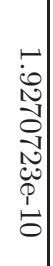 & 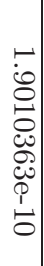 & 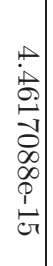 & 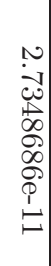 & 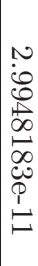 & 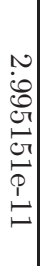 & 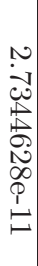 & 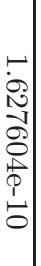 & 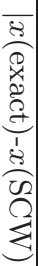 \\
\hline $\begin{array}{l}\circ \\
8 \\
8 \\
8 \\
8 \\
0 \\
\infty \\
\infty \\
0 \\
0 \\
0 \\
0 \\
0 \\
0\end{array}$ & 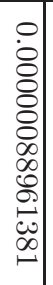 & 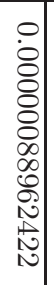 & 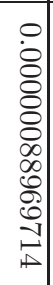 & 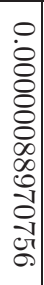 & 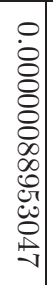 & $\begin{array}{l}0 \\
8 \\
8 \\
8 \\
8 \\
8 \\
8 \\
0 \\
8 \\
0 \\
\infty \\
0 \\
-1\end{array}$ & 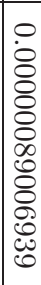 & 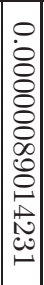 & 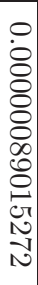 & 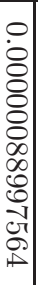 & 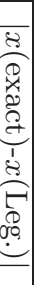 \\
\hline 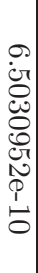 & 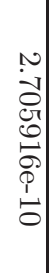 & $\begin{array}{l}1 \\
0 \\
0 \\
0 \\
\mathbb{1} \\
0 \\
\mathbb{1} \\
0 \\
0 \\
0 \\
0\end{array}$ & 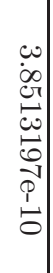 & $\begin{array}{l}0 \\
-1 \\
0 \\
0 \\
0 \\
0 \\
0 \\
0 \\
0 \\
0 \\
0\end{array}$ & $\begin{array}{l}0 \\
N \\
0 \\
0 \\
0 \\
0 \\
0 \\
0 \\
\stackrel{1}{0} \\
\dot{1}\end{array}$ & 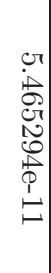 & 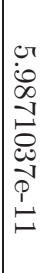 & 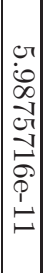 & 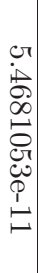 & 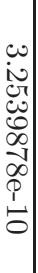 & 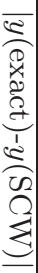 \\
\hline 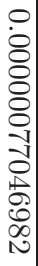 & 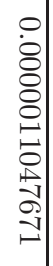 & $\begin{array}{l}0 \\
0 \\
8 \\
8 \\
8 \\
0 \\
0 \\
0 \\
0 \\
0 \\
0 \\
0 \\
0\end{array}$ & $\begin{array}{l}0 \\
0 \\
8 \\
8 \\
8 \\
0 \\
0 \\
-1 \\
\mathbb{N} \\
0 \\
0 \\
-1 \\
-1\end{array}$ & 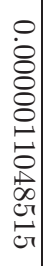 & $\begin{array}{l}0 \\
0 \\
8 \\
8 \\
8 \\
8 \\
0 \\
-1 \\
0 \\
0 \\
-1 \\
0 \\
0 \\
0\end{array}$ & 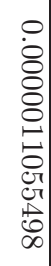 & 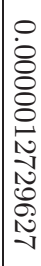 & 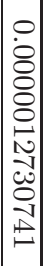 & 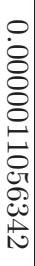 & 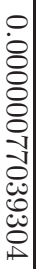 & 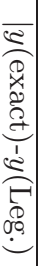 \\
\hline 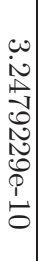 & 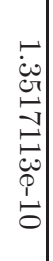 & 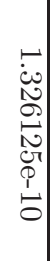 & 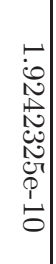 & $\begin{array}{l}- \\
0 \\
0 \\
0 \\
0 \\
0 \\
0 \\
0 \\
0 \\
1 \\
0\end{array}$ & 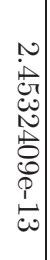 & $\begin{array}{l}0 \\
\\
0 \\
0 \\
0 \\
0 \\
0 \\
0 \\
0 \\
1 \\
\end{array}$ & 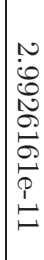 & $\begin{array}{l}1 \\
0 \\
0 \\
0 \\
0 \\
0 \\
0 \\
0 \\
0 \\
0 \\
1 \\
1\end{array}$ & 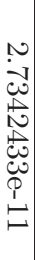 & 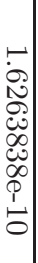 & 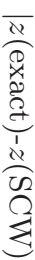 \\
\hline 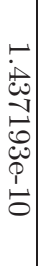 & 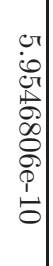 & 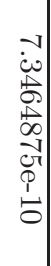 & 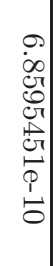 & $\begin{array}{l}c \\
-1 \\
1 \\
0 \\
0 \\
0 \\
0 \\
0 \\
1 \\
0 \\
0\end{array}$ & $\begin{array}{l}0 \\
i 0 \\
0 \\
\infty \\
0 \\
0 \\
0 \\
0 \\
0 \\
0 \\
0\end{array}$ & $\begin{array}{l}N \\
0 \\
0 \\
0 \\
0 \\
0 \\
. \\
0 \\
0 \\
1 \\
0 \\
0\end{array}$ & 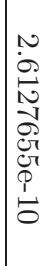 & 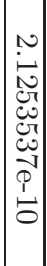 & 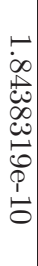 & 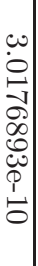 & 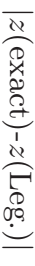 \\
\hline
\end{tabular}




$$
\begin{aligned}
& y^{\prime}(t)+z^{\prime}(t)=2 x(t) \\
& z^{\prime}(t)+x^{\prime}(t)=2 y(t)
\end{aligned}
$$

with the initial conditions given by

$$
x(0)=0, y(0)=0, z(0)=1
$$

The exact solution of above system of equations is given by

$$
\begin{aligned}
& x(t)=\frac{1}{3} e^{t}-\frac{1}{3} e^{-2 t} \\
& y(t)=\frac{1}{3} e^{t}-\frac{1}{3} e^{-2 t} \\
& z(t)=\frac{1}{3} e^{t}+\frac{2}{3} e^{-2 t}
\end{aligned}
$$

For numerical solution we use the following function approximation of $x^{\prime}(t)$, $y^{\prime}(t), z^{\prime}(t)$ in terms of second kind Chebyshev wavelet

$$
\begin{aligned}
x^{\prime}(t) & =C_{1}^{T} \Psi(t) \\
y^{\prime}(t) & =C_{2}^{T} \Psi(t) \\
z^{\prime}(t) & =C_{3}^{T} \Psi(t)
\end{aligned}
$$

Where $C_{1}^{T}, C_{2}^{T}, C_{3}^{T}$ are $2^{k-1} M \times 1$ matrices. Integrating from 0 to $t$ using operational matrix of integration, we obtain

$$
\begin{aligned}
& x(t)=C_{1}^{T} P \Psi(t) \\
& y(t)=C_{2}^{T} P \Psi(t) \\
& z(t)=C_{3}^{T} P \Psi(t)+d^{T} \Psi(t)
\end{aligned}
$$

Using the above results in given system, we get following system of equations

$$
\begin{aligned}
& C_{1}^{T} \Psi(t)+C_{2}^{T} \Psi(t)-2 C_{3}^{T} P \Psi(t)=2 d^{T} \Psi(t) \\
& C_{2}^{T} \Psi(t)+C_{3}^{T} \Psi(t)-2 C_{1}^{T} P \Psi(t)=0 \\
& C_{3}^{T} \Psi(t)+C_{1}^{T} \Psi(t)-2 C_{2}^{T} P \Psi(t)=0
\end{aligned}
$$

The above equations are satisfied for each $t$ in the defined interval. Therefore

$$
\begin{aligned}
& C_{1}^{T}+C_{2}^{T}-2 C_{3}^{T} P=2 d^{T} \\
& C_{2}^{T}+C_{3}^{T}-2 C_{1}^{T} P=0 \\
& C_{3}^{T}+C_{1}^{T}-2 C_{2}^{T} P=0
\end{aligned}
$$


These equations can be solved for vectors $C_{1}^{T}, C_{2}^{T}, C_{3}^{T}$. After finding $C_{1}^{T}, C_{2}^{T}$, $C_{3}^{T}$ we can get the approximate values of $x(t), y(t), z(t)$ by inserting these values in (7). The absolute error of exact and approximate solution for $M=3, k=2$ using second kind Chebyshev wavelet method and Legendre wavelet method is given in Table 3.

\section{Concluding Remarks}

In this paper we present a numerical method for approximating the solution of system of linear differential equations by combining second kind Chebyshev wavelet with their operational matrix of integration. The main advantage of the present method is its simplicity and small computation cost. Some examples are given to verify and demonstrate the effectiveness of the proposed method. Comparison of the absolute errors of proposed method with the Legendre wavelet method shows that this method is highly efficient and accurate.

\section{Acknowledgements}

The authors are thankful to the University Grant Commission, New Delhi for providing the financial assistance for the preparation of manuscript. 


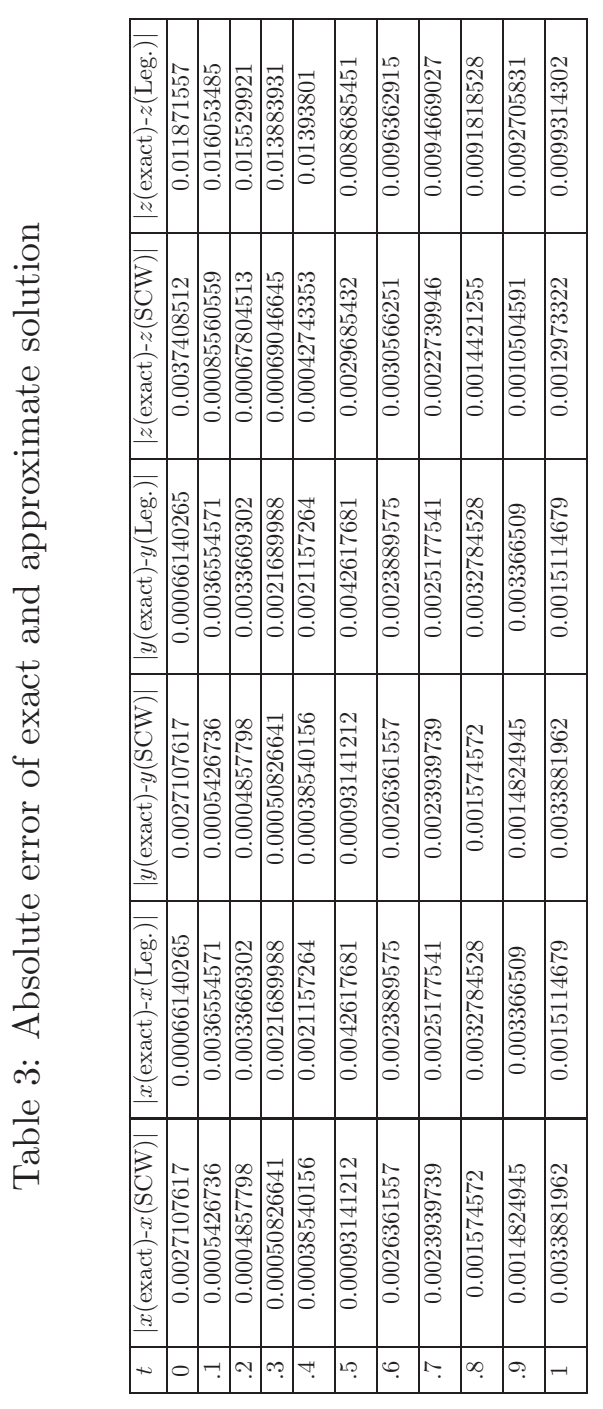




\section{References}

[1] E. Babolian, F. Fattahzadeh, Numerical solution of differential equations by using Chebyshev wavelet operational matrix of integration, Applied Mathematics and Computation 188 (2007), 417-426.

[2] Y. Wang, Q. Fan, The second kind Chebyshev wavelet method for solving fractional differential equations, Applied Mathematics and Computation 218 (2012), 8592-8601.

[3] J. Biazar, E. Babolian, R. Islam, Solution of the system of ordinary differential equations by Adomian decomposition method, Applied Mathematics and Computation 147 (2004), 713-719.

[4] N. Dogan, Solution of the system of Ordinary Differential Equations by combined Laplace Trnsfom-Adomain Decomposition Method, Mathematical and Computational Applications 17 (2012), 203-211.

[5] I.H. Abdel-Halim Hassan, Application to differential transformation method for solving systems of differential equations, Applied Mathematical Modelling 32 (2008), 2552-2559.

[6] He, J.H., Variational iteration method for autonomus ordinary differential systems, Applied Mathematics and Computation 114 (2000), 115-123.

[7] U. Lepik, Haar wavelet method for solving higher order differential equations, International Journal of Mathematics and Computation 1 (2008), 84-94.

[8] P. Manchanda, Mamta Rani, Non-Uniform Haar Wavelet Matrix Method for Numerical Solution of Ordinary Differential Equations, Indian Journal of Industrial and Applied Mathematics 6 (2015), 184-195.

[9] U. Lepik, Application of the Haar wavelet transform for solving integral and differential equations, Proceedings of the Estonian Academy of Sciences. Physics. Mathematics 56 (2007), 28-46.

[10] M. Razzaghi, S. Yousefi, Legendre wavelets direct method for variational problems, Mathematics and Computers in Simulation 53 (2000), 85-192.

[11] L. Zhu, Y. Wang, Second Chebyshev wavelet operational matrix of integration and its application in the calculus of variations, International Journal of Computer Mathematics 90 (2013), 2338-2352.

[12] L. Zhu, Q. Fan, Solving fractional nonlinear Fredholm integro-differential equations by the second kind Chebyshev wavelet, Communications in Nonlinear Science and Numerical Simulation 17 (2012), 2333-2341.

[13] L. Zhu, Y.X. Wang, Q.B. Fan, Numerical computation method in solving integral equation by using the second chebyshev method, In: The 2011 international conference on scientific computing. USA:Las Vegas 86 (2011), 126-130.

[14] I. Celik, Chebyshev wavelet collocation method for solving generalized Burgers-Huxley equation, Mathematical Methods in the Applied Sciences39 (2016), 366-377. 